\title{
A PERCEPÇÃO DE PROFESSORES SOBRE PROJETOS E ATIVIDADES ESPECIAIS IMPLEMENTADOS EM PARALELO ÀS DISCIPLINAS DO CURSO
}

Gabriel Pendloski Fraga - pendloski.fraga@gmail.com Instituto Mauá de Tecnologia - Curso de Engenharia Civil Praça Mauá, 01

CEP 09580-900 - São Caetano do Sul - São Paulo

Octavio Mattasoglio Neto-omattasoglio@maua.br Instituto Mauá de Tecnologia, Ciclo Básico

Praça Mauá, 01

CEP 09580-900 - São Caetano do Sul - São Paulo

Resumo: A implementação dos Projetos de Atividade Especiais (PAEs) no Instituto Mauá de Tecnologia (IMT) em 2015 tinha diversos objetivos a serem alcançados. Objetivos esses como a diversificação de conteúdos e as diferentes formas de aplicação, tornando o currículo do estudante mais completo e moldado da maneira que por ele for desejada. Após cinco anos da sua implementação, o objetivo deste trabalho é checar, pela percepção dos professores, quais desses objetivos foram atingidos. Foi criado um questionário em GoogleDocs que foi aplicado em professores de diversas áreas de engenharia do IMT. Os dados obtidos foram analisados e a partir de então foi possível esclarecer as dúvidas na qual nosso trabalho foi moldado. Na percepção dos professores, todos concordam que as PAEs são importantes, e que a mudança curricular foi positiva. Quanto ao amadurecimento dos alunos e o conhecimento deles sobre os reais objetivos das PAEs, apenas $12 \%$ dos respondentes disseram acreditar que os alunos estão cientes dos objetivos das reformas curriculares de cinco anos atrás.

Palavras-chave: PAEs. Aprendizagem Ativa. Ensino por Projetos. Avaliação de currículo. 


\section{INTRODUÇÃO}

Após o quinto ano do início da implantação de uma nova estrutura curricular, completouse o ciclo com a formatura da primeira turma desse curso, o que aconteceu no ano de 2019. Ao longo desse tempo a equipe de professores desse curso teve a responsabilidade de criar projetos e atividades especiais que complementaram a formação dos estudantes, promovendo o desenvolvimento de competências desejáveis pelos ambientes profissionais que empregam esses estudantes (MESQUITA, 2013), além de serem requeridas pelos profissionais que buscam empreender.

Os Projetos e Atividades Especiais (PAEs) são atividades que utilizam de metodologias diferentes de uma aula comum, existem PAEs direcionadas ao desenvolvimento acadêmico na tentativa de aprimorar os conhecimentos de uma aula do currículo regular, assim como outras direcionadas para o mercado, que preparam o estudante para diversas situações como por exemplo: uma entrevista num processo seletivo, a preparação de um currículo, o comportamento que deve ter numa possível negociação, como ser um líder em diversas ocasiões, conhecimentos sobre programação em diversos aplicativos fundamentais em muitas profissões, entre outros, direcionadas para o dia-a-dia, e ainda outros tipos, sempre envolvendo aprendizado e crescimento para os estudantes.

A opção de inclusão das PAEs no currículo dos cursos dessa escola foi motivada pela percepção de que os alunos deveriam se aproximar da Engenharia desde as séries iniciais do curso, resolvendo problemas e realizando projetos, que é uma vocação do engenheiro. Outra motivação foi o fato da escola ter um grande número de laboratórios que poderiam ser melhor utilizados pelos estudantes, alinhando-se com a primeira motivação.

As PAEs podem ser escolhidas pelos estudantes, dessa forma é possível aprender sobre assuntos de sua preferência e assim adquirir conhecimentos e habilidades necessários em uma área específica de atuação. O estudante constrói sua trilha de aprendizado, podendo, por exemplo, no seu curso de engenharia buscar um caminho mais voltado para o empreendedorismo, ou um caminho mais voltado à pesquisa, ou um caminho mais voltado para o desenvolvimento de habilidade técnicas profissionais ligada diretamente à sua área de formação. Como premissa as PAEs não realizam provas ou testes para a aprovação do estudante, que deve ser essencialmente estabelecida pela participação nas atividades.

O objetivo deste trabalho é compreender a percepção dos professores sobre o andamento das PAEs desde que foram introduzidas no Instituto Mauá de Tecnologia (IMT) cinco anos atrás, as alterações consideradas como positivas e aquelas que merecem revisão. Cabe lembrar que a mudança curricular realizada atendia na época as DCNs para os cursos de engenharia (MEC, 2002) e hoje atende as atuais DCNs para esses cursos (ABENGE, 2019).

A realização desse trabalho é importante para melhor percepção do IMT sobre o que poderia ser melhorado, e se cinco anos após a reforma curricular na qual foi instaurada a utilização desse novo elemento no currículo, ainda restam dúvidas de suas funções e utilidades. Essas respostas, podem levar a mudanças que solucionem alguns problemas constatados.

Após a realização de uma revisão bibliográfica, criou-se um questionário que foi aplicado em alguns professores selecionados de todos os cursos do IMT. A partir dos dados obtidos, realizou-se uma análise e se chegou a algumas conclusões sobre as percepções dos professores sobre as PAEs. 


\section{REVISÃO BIBLIOGRÁFICA}

Neste trabalho relativamente à avaliação da implementação da uma reforma curricular, dois pontos são importantes a serem considerados na revisão bibliográfica, e serão apresentados aqui.

\subsection{Criação das PAEs no contexto de uma reforma curricular}

Uma reforma curricular que visa a implantação do PBL tem como sustentação 3 pontos: infraestrutura, autoridade e consenso (POWELL \& WEENK, 2003). A dimensão "infraestrutura" envolve instalações como por exemplo salas de aula e laboratórios formação de professores e comunicação. $\mathrm{O}$ último estabelece uma base comum em relação à percepção e à necessidade de mudança. É necessária autoridade para promover planejamento, orientação e progressão adequados para garantir que a implementação seja aceita e institucionalizada. Por outro lado, partilha de informações e experiências e o comprometimento e visão de professores com foco na aprendizagem dos alunos atribuem uma característica bottom-up para o projeto curricular. Por fim, o consenso facilita a identificação de problemas cruciais para o sucesso do PBL, com a inclusão de partes interessadas no processo de inovação. "Cooperação entre os professores envolvidos no PBL é tão essencial quanto a cooperação entre estudantes na equipe deles" (POWELL \& WEENK, 2003).

O desenvolvimento do currículo envolve três etapas: preparação, implementação e avaliação. É um processo coletivo, que inclui pessoas e procedimentos, envolvimentos interpessoais, políticas e dimensões sociais, além da colaboração e cooperação dos envolvidos. Não é um processo científico racional, devido à subjetividade envolvido, nem um processo linear ou sistematizado. As diretrizes subjetivas e seus recursos flexíveis fornecem ao desenho curricular um grau de abertura (PACHECO, 2005).

Desde a concepção até sua completa implementação, o currículo passa por diferentes níveis. Para Goodlad (1979) o ponto de partida de um currículo é o 'currículo ideal'. Depois dessa etapa inicial, existe o "currículo formal", que é revelado nos documentos curriculares, como manuais e livros didáticos, e se traduz no currículo oficial. O terceiro passo é o "currículo operacional", desenvolvido por um grupo ou indivíduo único, que traduz as ideias e formalismo definido na etapa anterior para o cotidiano (prática). Por fim, existe o "currículo percebido", que é experimentado em atividades diárias da sala de aula, o qual pode ser observado hoje em dia, no Instituto Mauá de Tecnologia. Há também o "currículo avaliado", que inclui avaliação do aluno e de currículo planos, programas, diretrizes, manuais e livros didáticos, professores, escola e administração.

A fase de implementação das PAEs nesta escola de engenharia foi estudada por Mattasoglio Neto et al. (2019) que indicaram como ponto principal a necessidade de melhorar a comunicação aos estudantes sobre o propósito das PAEs. Os autores indicaram que a comunicação vai ao encontro do consenso entre professores e alunos e a falha nessa comunicação que pode comprometer a implantação da nova proposta.

\subsection{A avaliação nas PAEs}

Como observou Macedo et al (2017), num trabalho sobre PAEs vinculado à área de Engenharia Mecânica, o processo de avaliação foi desenvolvido ao longo do curso, confirmando o traço forte do método PBL. "Porém, muitos alunos criticaram a excessiva carga horária que as atividades demandam e a quantidade de trabalhos exigidos, principalmente porque as datas de entrega coincidiram com as semanas de provas". 
Estabelecer um framework para a introdução de projetos (LIMA et al, 2012) num curso é importante porque pode dar subsídios para reformulações e reflexões, por exemplo na estratégia de avalição desses cursos. Nesse sentido, há que se verificar se esse framework foi alcançado após o fechamento dessa etapa de implantação da introdução das PAEs.

\section{MÉTODO DE PESQUISA}

Para avaliação da percepção dos professores do Instituto Mauá de Tecnologia - IMT sobre as PAEs foi elaborado um questionário alinhado como os propósitos iniciais do trabalho. A opinião dos professores daria subsídios para comparar com a percepção atual dos professores com aquela do início do processo de implantação das PAEs em 2015. Esse conhecimento e essa comparação deveria permitir a melhoria de maneira geral das PAEs oferecidas do IMT.

Depois de encaminhar as perguntas para treze professores de diversas áreas de engenharia do Instituto Mauá de Engenharia, oito responderam o questionário, a partir do que se pode organizar os dados relevantes para este trabalho. Os dados foram obtidos a partir da ferramenta Google.docs e organizados numa planilha Excel, o que permitiu a filtragem e separação dos dados voltados para a perguntas que se desejava obter resposta.

Foram consideradas as seguintes dimensões de análise neste trabalho: Caracterização da amostra; A importância das PAEs na visão dos respondentes; Como os alunos sentem as PAEs na visão dos professores; Avaliação nas PAEs; Diferença percebida entre as PAEs e as disciplinas do currículo. Os dados e sua análise, segundo essas dimensões estão, apresentados no item 4 deste trabalho.

\section{RESULTADOS DO QUESTIONÁRIO}

A análise dos dados e a discussão estão indicadas nos itens que seguem.

\subsection{Caracterização da amostra}

Como se pode observar na Figura 1 e Figura 2 a maioria dos respondentes são do sexo masculino e na faixa etária de 40 a 50 anos.

Figura 1 - Sexo dos respondentes

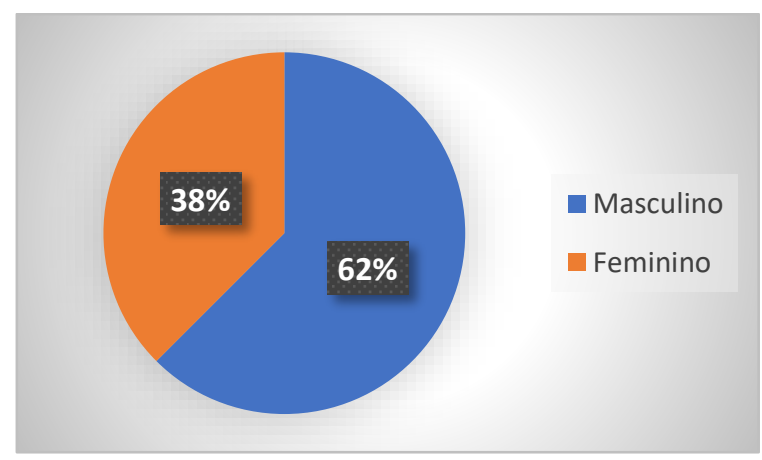

Fonte: Os autores
Figura 2 - Faixa etária

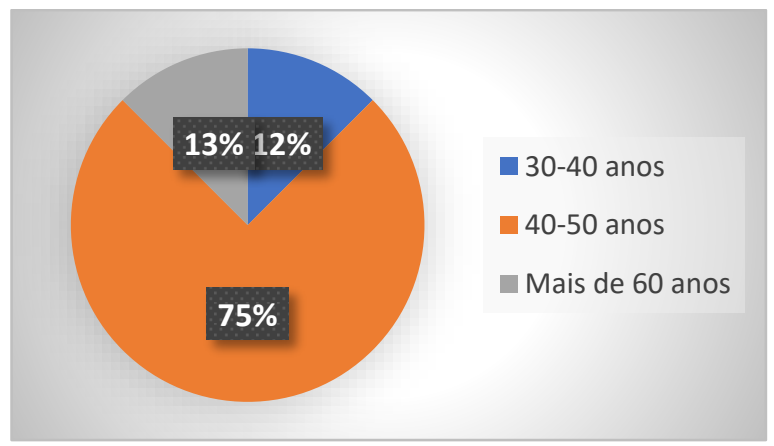

Fonte: Os autores

Todos os respondentes, independentemente do tempo de docência no IMT (Figura 4), concordam sobre a importância do uso de estratégias ativas no aprendizado (Figura 3), que são aquelas que fundamentam as PAEs. 
Figura 3 - Importância das estratégias ativas

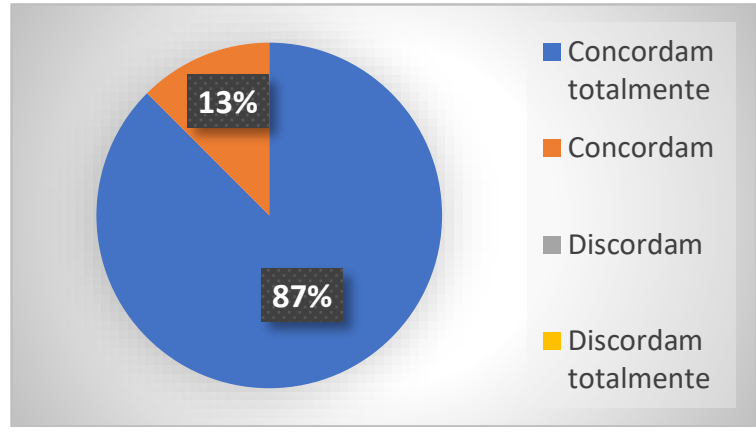

Fonte: Os autores
Figura 4 - Tempo de docência no IMT

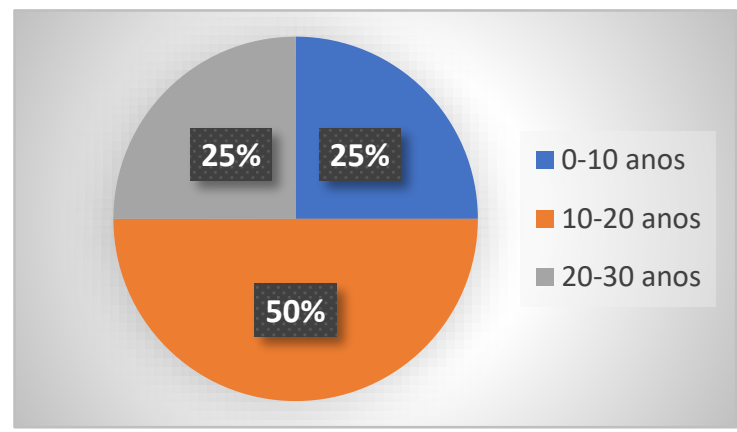

Fonte: Os autores

\subsection{A importância das PAEs na visão dos respondentes}

Todos os respondentes (100\%) veem nas PAEs uma mudança positiva e geradora de grande melhoria aos cursos do IMT, porém não são todos (13\%) que acreditam que as PAEs que não estão diretamente ligadas a conteúdos técnicos de engenharia são importantes (Figura 5). A percepção da grande maioria de que mesmo PAEs não diretamente ligada às disciplinas do curso são importantes indica a aceitação de que existem competências além daquelas técnicas que devem ser desenvolvidas pelos estudantes, como as competências desejáveis pelo mercado de trabalho e relacionadas a aspectos comportamentais, por exemplo.

Figura 5 - Importâncias das PAEs não diretamente relacionadas ao curso

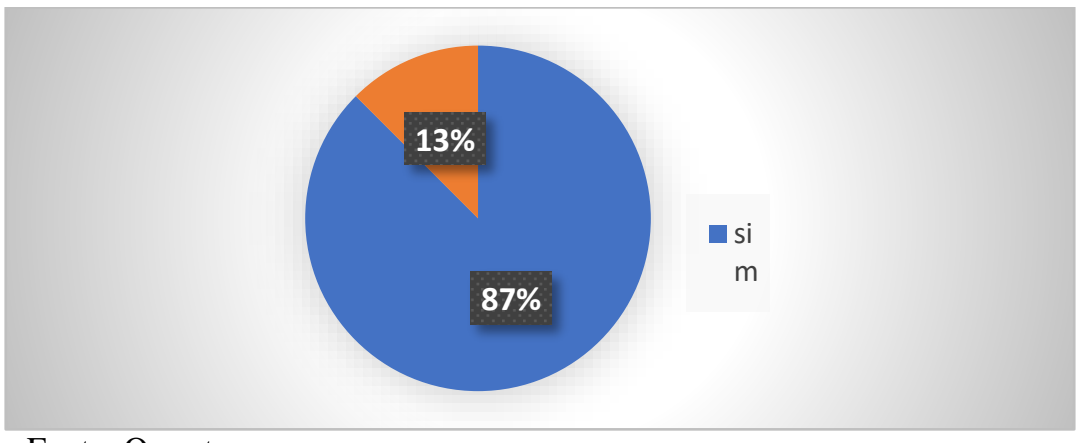

Fonte: Os autores

\subsection{Como os alunos sentem as PAEs na visão dos professores}

A questão que gerou maior discussão foi sobre o amadurecimento dos alunos em relação ao entendimento sobre o valor agregado pelas PAEs aos seus currículos e sua importância de forma geral (Figura 6), e junto disso $75 \%$ dos professores têm dúvidas sobre a ciência do aluno referente a proposta curricular que as PAEs representam (Figura 7). 
Figura 6 - Percebe nos alunos amadurecimento para entender a importância das PAEs

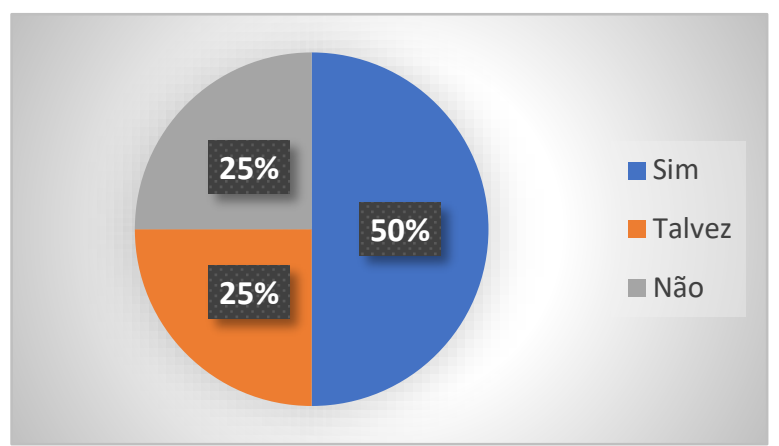

Fonte: Os autores
Figura 7 - O aluno está ciente dos objetivos da proposta curricular de 5 anos atrás

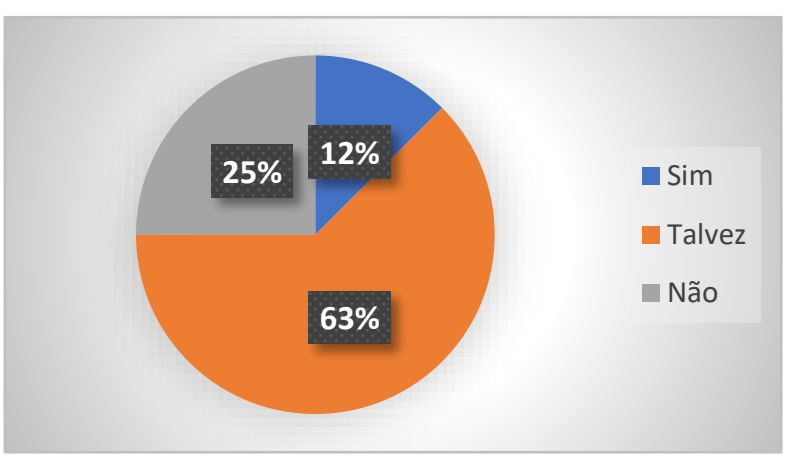

Fonte: Os autores

O fato de os alunos não saberem da importância do aprendizado ativo, e não terem tantos conhecimentos sobre a proposta curricular que a reforma de cinco anos atrás gerou, faz com que alguns, na percepção dos professores, não levem as PAEs tão a sério e só cumpram suas horas anuais obrigatórias para serem aprovados.

Essa percepção sobre o amadurecimento dos estudantes para o novo currículo e para as PAEs já era observado por Mattasoglio et al. (2019), quando analisou a implantação das PAEs em 2015. Esse mesmo resultado indica a necessidade de uma ação voltada especificamente para superar esse cenário. Uma sugestão é um trabalho de divulgação do propósito das PAEs e o alinhamento entre a percepção de alunos e professores.

\subsection{Professores aplicadores de PAEs}

Dos professores entrevistados 75\% eram aplicadores de PAEs (Figura 8) e todos estão satisfeitos com as PAEs que ministram.

Figura 8 - Professores entrevistados aplicadores de PAE

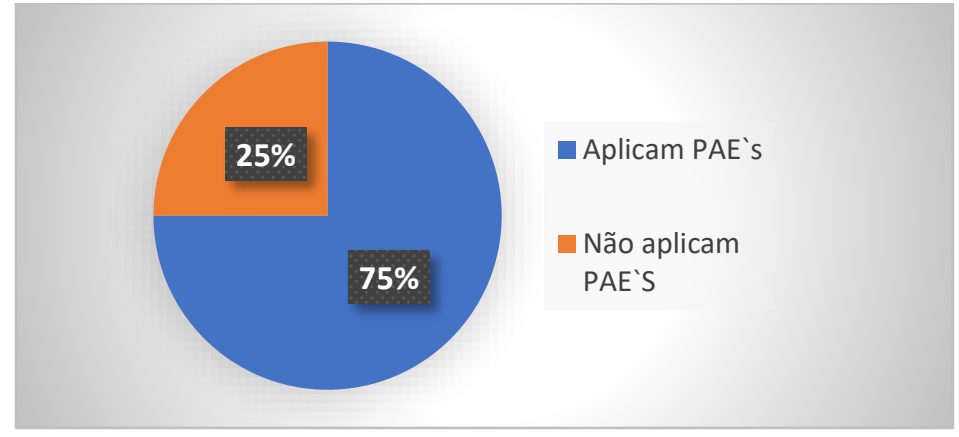

Fonte: Os autores

\subsection{Avaliação da aprendizagem nas PAEs}

Dos professores respondentes $83 \%$ não realizam nenhum tipo de avaliação nas suas PAEs, no entanto, esse mesmo número de professores é o dos que dão aos alunos um feedback ao término das PAEs Figura 9 e Figura 10.

As PAEs têm como premissa não realizarem avaliação do aprendizado dos estudantes, no entanto, o feedback sobre o desenvolvimento de competências é fundamental para que o estudante perceba o quanto se aproveitou no desenvolvimento de competências essenciais relacionadas ao curso que realizou. 
Figura 9 - Realização de avaliação nas PAEs

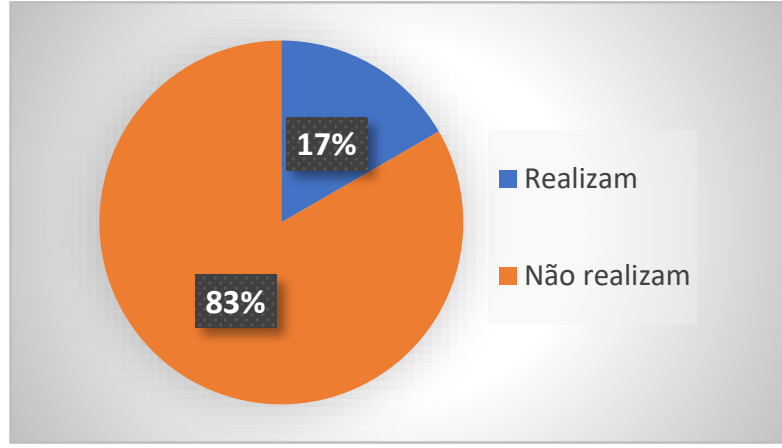

Fonte: Os autores
Figura 10 - Realização de feedback ao término da PAEs

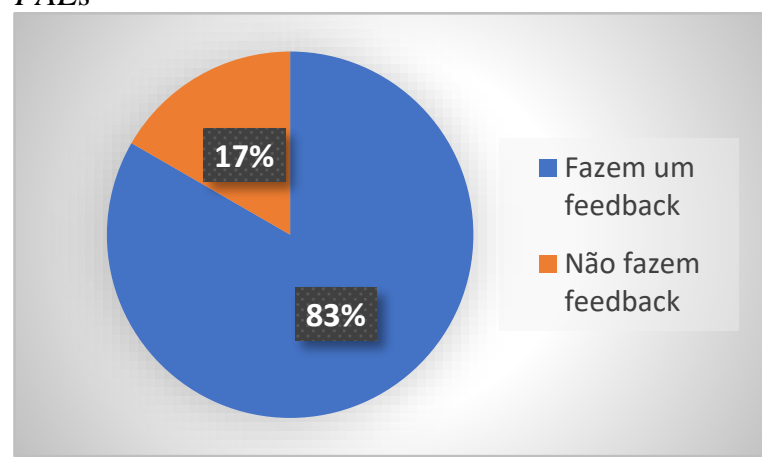

Fonte: Os autores

A grande maioria dos professores (56\%) usa como método de avalição, para a aprovação dos alunos, o comprometimento dos mesmos durante o desenvolvimento da PAE, já 33\% utilizam outros métodos de avaliação, $11 \%$ não realizam nenhum tipo de avaliação e a aprovação dos alunos apenas depende da sua presença nas aulas. Nenhum dos professores respondentes realizam uma prova/teste que determinaria a aprovação dos alunos (Figura 11).

Figura 11 - Métodos de avaliação

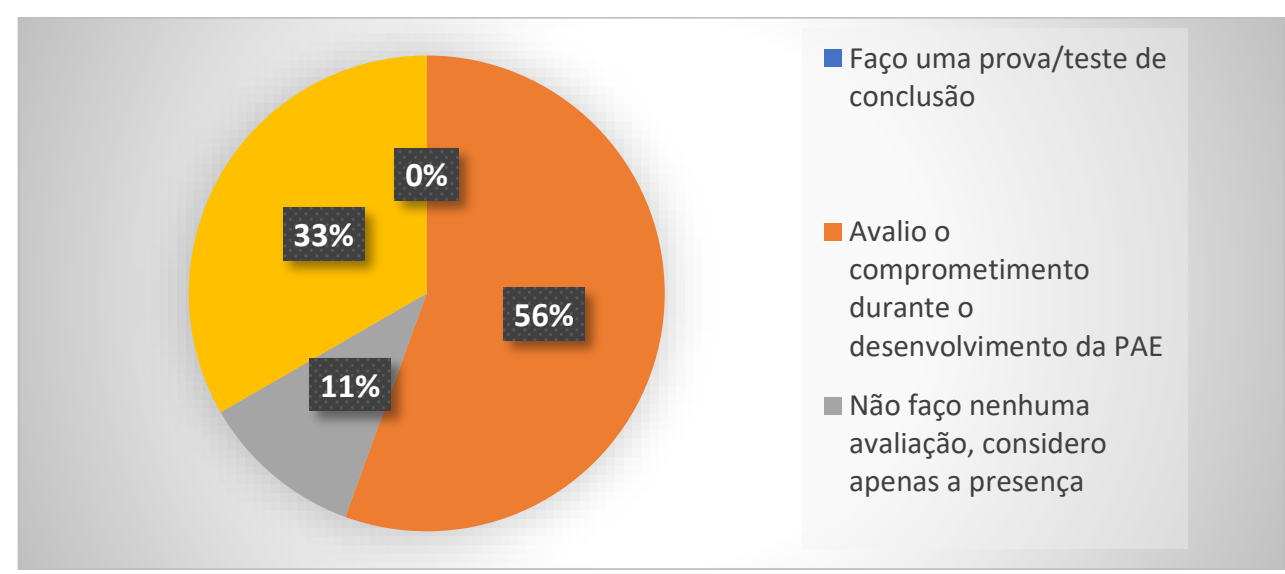

Fonte: Os autores

A não realização de provas nas PAEs é uma diretriz da escola, no entanto há a percepção de que há necessidade de se criar instrumentos para acompanhar o desenvolvimento das competências que se deseja alcançar. Não foi investigado a existência desses instrumentos nem foi espontaneamente declarado pelos respondentes o uso de qualquer tipo de instrumento para então ser realizado o feedeback sobre o desenvolvimento dos estudantes.

\subsection{Diferenças entre PAEs e disciplinas do curso}

Para os respondentes a principal diferença entre o trabalho conduzido numa PAE e uma aula normal é a interação dos alunos "A flexibilidade e interação dos alunos" (Respondente 1), multidisciplinariedade que essas aulas promovem, interdisciplinaridade e principalmente a prática. "Enquanto aula seria mais teoria a PAE é totalmente prática" segundo um dos entrevistados (Respondente 3). 
O fato de a PAE promover a prática está diretamente relacionado com a concepção inicial das PAEs, no currículo idealizado, que indicava a tentativa de fazer mais uso dos laboratórios da escola e aproximar os alunos do modo de fazer do engenheiro que pressupõe o "mão na massa", ou seja, efetivamente praticar. A flexibilização é algo que também aparecia na proposta inicial, e que hoje é ainda mais valorizada nos currículos de engenharia.

\subsection{Análise dos dados}

Todos os professores concordam que as PAEs são importantes, mas para os respondentes, apenas um não percebe em PAEs, que não estão diretamente relacionadas ao curso, essa importância. A pergunta que gerou maior divergência de opiniões foi quanto ao amadurecimento dos alunos e o conhecimento deles sobre os reais objetivos das PAEs tanto para conhecimentos pessoais quanto para aprimoramento dos currículos. Apenas (12\%) dos professores disseram acreditar que os alunos estão ciente dos objetivos das reformas curriculares de cinco anos atrás, em paralelo a isso observa-se que nenhum professor aplica prova de conclusão, sendo que a grande maioria (56\%) avalia o comprometimento do aluno durante o desenvolvimento da PAE e $11 \%$ apenas verifica presença, enquanto $33 \%$ utilizam outros métodos.

\section{CONSIDERAÇÕES FINAIS}

Os resultados obtidos com o questionário foram adequados com a proposta inicial do projeto. Esses resultados indicam que a opinião dos professores não mudou em relação a percepção dos alunos sobre as PAEs, os participantes da pesquisa continuam acreditando que os alunos não sabem a verdadeira importância das PAEs e da mudança curricular e só se preocupam em passar de ano, o que vai ao encontro do resultado da pesquisa realizada no primeiro ano de implantação da proposta curricular em 2015, quando os professores revelaram que os alunos não têm o nível desejado de conscientização sobre a importância das novas abordagens na sua formação, e consideravam PAEs como tarefas a serem cumpridas para obter uma nota de aprovação, em vez de serem percebidas como oportunidades de aprendizagem para o desenvolvimento de competências (MATTASOGLIO et al., 2019).

Por outro lado, nota-se diferença na opinião dos professores em relação aos assuntos que devem ser abordados nas PAEs. No artigo de 2019 vê-se que professores e alunos acreditavam que as PAEs deveriam abordar assuntos relacionados às disciplinas do curso, e na pesquisa realizada neste trabalho, pode-se observar que $87 \%$ dos professores acreditam na importância de PAEs não diretamente ligadas às disciplinas do curso.

Pode-se afirmar que o currículo percebido ainda não desenvolveu identidade em relação em relação ao currículo idealizado. Isso indica que ainda há a necessidade de uma "melhor comunicação com os alunos sobre os objetivos das PAEs, esclarecendo sua contribuição para o desenvolvimento de competências".

Se o currículo é um processo co-construído, a avaliação, tal como foi ensaiada neste trabalho é algo que deve continuar a ser realizado, dando subsídios para o aprimoramento desse currículo. Nesse sentido, algumas questões merecem um novo levantamento de dados para se avançar no entendimento das PAEs. Uma questão é como é dado o feedback aos estudantes e o que se espera com esse feedback. A importância da resposta dessa questão está no fato de o feedback poder dar indicação daquilo que o professor está valorizando na formação do estudante, a ponto de ser abordado nesse momento final do projeto. 


\section{Agradecimentos}

Ao CNPq pelo auxílio de bolsa iniciação científica.

Aos professores que gentilmente colaboraram respondendo a pesquisa.

\section{REFERÊNCIAS}

ABENGE - Proposta de parecer e de resolução para as DCNs Engenharia.

http://portal.mec.gov.br/index.php?option=com_docman\&view=download\&alias=109871pces001-19-1\&category_slug=marco-2019-pdf\&Itemid=30192 Accesso em 03 abril 2020.

GOODLAD, J. Curriculum inquiry: The study of curriculum practice, McGraw-Hill, New York, 1979

LIMA, R. M., CARVALHO, D., SOUZA, R. M. A. da S. e, ALVES, A., MOREIRA, F., MESQUITA, D., FERNANDES, S. A Project management framework for planning and executing interdisciplinary learning projects in engineering education. In: Project approaches to learning in engineering education. (2012) CAMPOS, L. C. de, DIRANI, E. A. T., MANRIQUE, A. L. and HATTUN-JANSSEN, N. van. Rotterdan, Sense Publisers. 2012.

MACEDO, N. D.; MATTASOGLIO Neto, O. A percepção de estudantes e professores da segunda série de engenharia sobre projetos e atividades especiais com ênfase em Engenharia Mecânica. Anais: XLV - Congresso Brasileiro de Educação em Engenharia. Joinville: ABENGE, 2017.

MATTASOGLIO Neto, O.; LIMA, R. M.; MESQUITA, D. Changing an Engineering Curriculum through a Co-Construction Process: A Case Study. International Journal of Engineering Education. V. 35, n. 4, pp 1129-1140. 2019.

MEC - Ministério da Educação e Cultura. Conselho Nacional de Educação. Câmara de Educação Superior. Diretrizes Curriculares para os cursos de engenharia. Resolução CNE/CES nº 11, de 11 de março de 2002.

MESQUITA, D., LIMA, R. M., \& FLORES, M. A. Developing professional competencies through projects in interaction with companies: A study in Industrial Engineering and Management Master Degree. Proceedings: Fifth International Symposium on Project Approaches in Engineering Education, PAEE'2013. Eindhoven, The Netherlands. 2013. Actas: PAEE'2013. Eindhoven, The Netherlands. 2013.

PACHECO, J. A. Escritos curriculares. Cortez, São Paulo, p. 176, 2005.

POWELL, P. C., WEENK, W. Project-led engineering education. Lemma, Utrecht, 2003. 


\title{
THE TEACHER'S PERCEPTION OF SPECIAL PROJECTS AND ACTIVITIES IMPLEMENTED IN PARALLEL TO THE COURSE DISCIPLINES
}

\begin{abstract}
The implementation of the Project of Special Activities, known as PAE's at IMT, the Maua Institute of Technology in 2015 had several goals to achieve, such as the diversification of content and its different ways of application, turning the curriculum of the students complete and shaped in their desired manner. Over the course of five years of the PAE's implementation, we came through this to verify using professor's perception to know which of these goals mentioned above were reached. A GoogleDocs questionnaire was created and sent by professors in different engineering fields of IMT. The data obtained was analyzed and from that it was possible to clarify all the doubts in which our survey was based. On the perception of our professors surveyed, it's agreed that the PAE's have great importance and its curricular change was positive. In relation with the academic growth of students and their knowledge about the real objective of the PAE's, only $12 \%$ of professors come to believe that students are aware of the goals and objectives of the curricular alterations from the past 5 years.
\end{abstract}

Key-words: PAEs. Active Learning. Teaching by project. Curriculum evaluation. 